\title{
MICROFABRICATED PROBES FOR LABORATORY PLASMAS
}

\author{
Janet A. Stillman ${ }^{1}$, Franklin C. Chiang ${ }^{1}$, Patrick A. Pribyl ${ }^{2}$, Mio Nakamoto ${ }^{2}$, \\ Walter Gekelman ${ }^{2}$, and Jack W. Judy ${ }^{1}$ \\ Departments of Electrical Engineering ${ }^{1}$ and Physics ${ }^{2}$, University of California at Los Angeles, Los Angeles, \\ California, 90095, USA
}

\begin{abstract}
We present the first of a series of microfabricated sensors for directly measuring fundamental plasma parameters with unprecedented resolution in typical laboratory plasmas. Microfabricated probes hold the promise of significantly advancing basic plasma physics by enabling the measurement of some fundamental plasma processes under controlled conditions at the relatively high plasma densities typically of interest. Our first probes are arrays of micrometer-scale electric-field sensors for directly measuring fluctuating electric fields in the Large Plasma Device (LAPD) at UCLA. They are made of polyimide, chrome, and gold, have tip widths ranging from $8 \mu \mathrm{m}$ to $20 \mu \mathrm{m}$, are $22 \mu \mathrm{m}$ thick, and spaced $40 \mu \mathrm{m}$ apart. The fabricated tips are wirebonded to a preamplifier PCB with commercial amplifiers, and everything is inserted inside a plasma chamber. The frequency response of the detectors extends to $1 \mathrm{GHz}$.
\end{abstract}

\section{INTRODUCTION}

Plasma, or ionized matter, supports a variety of collective modes and makes up a very large fraction (perhaps 99\%) of the observable universe. As the "fourth state of matter", plasmas also play a critical role in our economy, from the plasma processing of semiconductor wafers to the use of thermonuclear plasmas in energy research. In addition, low-temperature plasmas are already used in lighting, plasma-screen televisions, and in medicine for sterilization and dental procedures. However, despite the broad interest, utility, and widespread presence of plasmas, there are a great many fundamental unknowns about plasmas. This lack of fundamental plasma-science knowledge limits our ability to understand plasma behavior and to use it optimally for a wide range of economically significant applications (e.g., IC and MEMS manufacturing, etc.). Specifically, we need diagnostic tools and technologies that can operate at the smallest scales of import in plasmas.

One fundamental plasma parameter is the Debye length. Since a plasma is a collection of charged particles, introducing a charged object into the plasma causes plasma particles to move in response, with oppositely charged particles being attracted to the object. The net effect is that any electric fields from the object are shielded out of the bulk of the plasma by the plasma itself. The characteristic physical scale on which this happens is the Debye length, and beneath this size scale, a material object does not disturb the macroscopic plasma. The Debye length is given by

$$
\lambda_{D}=\sqrt{\frac{\varepsilon_{\mathrm{o}} \cdot k_{\mathrm{b}} \cdot T_{\mathrm{e}}}{n_{\mathrm{e}} \cdot q_{\mathrm{e}}^{2}}},
$$

with permittivity of free space $\varepsilon_{0}$, Boltzmann's constant $k_{\mathrm{b}}$, electron temperature $T_{\mathrm{e}}$, density of electrons $n_{\mathrm{e}}$, and electron charge $q_{\mathrm{e}}$. A plot of the Debye length as a function of the density of electrons in a plasma is given in Fig. 1 for a range of electron temperatures, and approximate parameter values are indicated for a variety of natural and human-made plasmas. An important observation from Fig. 1 is that the Debye length of human-made plasma systems is generally less than $1 \mathrm{~mm}$, whereas it is considerably larger than $1 \mathrm{~mm}$ for natural plasmas.

Much work has been done in using probes to diagnose plasmas. In space, where the Debye length can be meters, it is easy to construct probes of the right size, but it is extremely difficult to control experimental conditions. The situation is reversed for almost all terrestrial plasmas of interest, such as those used in the semiconductor and microfabrication industry or in basic laboratory research, with the physical dimensions of probes being larger than a Debye length, which is typically 30 to $100 \mu \mathrm{m}$ in these plasmas. Objects larger than these dimensions, when introduced into the plasma, result in a sheath in the local plasma potential surrounding them, and the physics of this sheath must be taken into account when attempting to deduce plasma properties from probe signals [1]. The presence of this sheath is particularly troublesome when attempting to measure fluctuating plasma electric fields. Such problems can sometimes be bypassed using non-invasive optical techniques $[\mathbf{2}, \mathbf{3}]$, but these techniques lack 3-D spatial resolution, are time consuming, and are very expensive. A better solution is to use appropriately small direct-measurement tools.

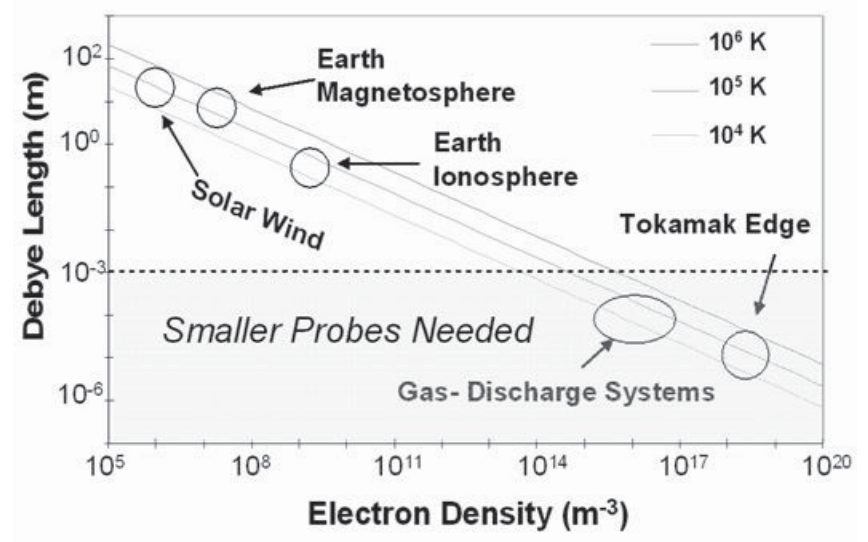

Fig. 1. Plot of the Debye length in a plasma as a function of the density of electrons for a range of electron temperatures.

Integration of microfabrication techniques with plasma research have already begun. Researchers, hoping to improve process control for the semiconductor industry, have successfully measured ion-flux distributions in plasma-etching reactors [4] and have begun integrating sensors into wafers for localized temperature measurements within plasma chambers [5]. However, all prior work used devices that were larger than the Debye length in the plasma being studied, which means there may be effects that have not been taken into account in the results.

Micromachining techniques have also been used to create microplasmas for localized etching and deposition [6]. Conventional machining techniques have yielded electric-field microprobes that showed promise in observing micron-scale fundamental phenomenon within plasmas [7], but were limited by the non-uniform spacing and angles between individual probe tips. The micromachined probes presented here overcome these shortcomings.

\section{MOTIVATION}

With the advent of micromachining and microelectromechanical-systems (MEMS) technologies, a variety of microscale sensors can be fabricated to measure high-density terrestrial plasmas without disturbing the plasma itself. These plasma-measurement devices could bring about a sea change in our 
ability to measure fundamental plasma parameters. The ultimate goal of this work is to further the understanding of plasma physics and plasma interactions in high-density plasma-processing tools. We will do this by creating a variety of microsensor systems (e.g., electric-field detectors and ion-energy analyzers).

\section{DESIGN}

Microscale measurement of the electric field requires differential wire tips sized on the order of the Debye length. Since the Debye length in the LAPD is typically 20 to $100 \mu \mathrm{m}$, we designed probe tip widths ranging from $8 \mu \mathrm{m}$ to $20 \mu \mathrm{m}$ and the two tips of a single pair spaced from $20 \mu \mathrm{m}$ to $52 \mu \mathrm{m}$ apart (Fig. 2). Tips of adjacent pairs are separated by $40 \mu \mathrm{m}$ to $108 \mu \mathrm{m}$, giving us the ability to track traversing signals.

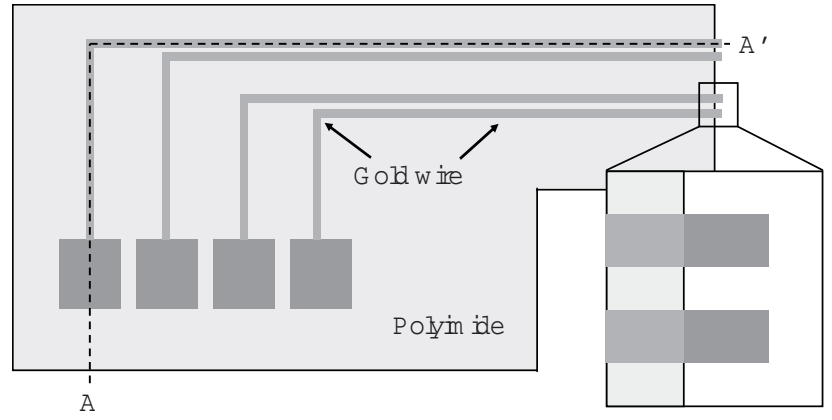

Fig. 2. Top view of the micromachined electric-field probe.

We arrayed several sets of differential pairs in a line in order to measure the electric field at several points along the magnetic field. We also interspersed probe-tip pairs with wire pairs lacking exposed tips, allowing us the opportunity to compare signals from the tips to signals from the wiring or other sources. Most of the probes are designed to measure the electric field along the magnetic field of the chamber, but we also included pairs for measuring the electric field perpendicular to the magnetic field.

We first tried using a high-deposition-rate PECVD oxide for mechanical support and insulation. However, after encountering many problems with intrinsic stress, we instead embedded the probes and wires in a $23-\mu \mathrm{m}$-thick cantilever of HD Microsystems PI-2600 low-stress polyimide. This polyimide has an advertised glass-transition temperature of $350{ }^{\circ} \mathrm{C}$ and does not begin to physically degrade until $620^{\circ} \mathrm{C}$. It is also mechanically stiff for a polyimide, with an elastic modulus of $8.5 \mathrm{GPa}$. In our process and handling we have found it nearly indestructible, and our yield was close to $100 \%$. With a mass density of about $1.4 \mathrm{~kg} / \mathrm{m}^{3}$, our cantilever/probe structure in vacuum had an estimated resonance frequency of about $4.8 \mathrm{kHz}$, well below the frequencies of interest.

With a relative permittivity of 2.9 , a $10-\mu$ m-thick layer of this polyimide is sufficient to yield a capacitance coupling of the 1-cm-long, 32- $\mu \mathrm{m}$-wide probe wire to the ambient plasma of less than $3 \mathrm{pF}$. This significantly reduces the coupling of the wires to the plasma as compared to the probe tips, and is still sufficiently thin relative to a Debye length.

We selected gold as the material for the electrodes, as it is easily wirebonded to. In addition, unlike aluminum, gold forms no significant oxide layer and so would be easier to model in the future. Gold also has a low magnetic permeability, is ductile, and has a low enough resistivity that we can achieve a sufficiently low resistance in 1-cm-long, $0.5-\mu \mathrm{m}$ thick, and 20- to 32- $\mu \mathrm{m}$-wide wires. We found that we could not wirebond to a thin layer of gold with our available wedge wirebonder, because the wirebonder tip punctured the gold pad on the flexible polyimide base without bonding to it. Our final process, therefore, involved evaporating a thin seed layer of gold and electroplating additional gold to a thickness of $2.5 \mu \mathrm{m}$.

\section{FABRICATION}

\section{A. Pre-amplification Circuitry and Housing}

We fabricated the preamplification circuitry with a custom 4-layer PCB using a 1.8-GHz commercial amplifier (Texas Instruments THS4303). The plasma normally has a potential of several tens of volts, so we capacitively couple the probe signal through a simple one-pole high-pass $\mathrm{RC}$ filter with a 3-dB frequency of about $5 \mathrm{MHz}$. We attempted to minimize the parasitic capacitance of the input circuit of this amplifier, with a target of less than $5 \mathrm{pF}$, and a resistance of less than $25 \Omega$.

A small custom-built copper box protects the electronics from the plasma. For cooling purposes, a copper tube with a hairpin turn is inserted into the feedthrough shaft to a point near the copper box and pressurized air is forced through the tube. The copper box has a screw-on lid and small holes on the front and back sides. Signal wires enter the box from the shaft through the hole on the back end, where they are soldered onto the PCB. We glued the probe chip onto the opposite end of the PCB, so that it stuck out through the front hole into the plasma. We then wirebonded the probe connections to the PCB.

The dimensions of the custom-built copper box are $7 \mathrm{~cm} \times 5 \mathrm{~cm} \times 0.5 \mathrm{~cm}$, which are large enough to cause a local disturbance to the plasma. We extended the probe tips as far as possible from the box to minimize these effects; for the initial test that distance was only $0.5 \mathrm{~cm}$ (about 200 times the Debye length); the next iteration will be a centimeter or more.

\section{B. Microprobe Fabrication}

The two-mask fabrication process for the probe tips begins with a silicon wafer whose only purpose is to provide a platform for the probe fabrication. As seen above in Fig. 3(a), a 10- $\mu$ m-thick film of polyimide (HD Microsystems PI-2600 LX) is deposited to form the lower insulator and the base of the mechanical support for the probe tips. A seed layer consisting of chrome $(10 \mathrm{~nm})$ and gold $(30 \mathrm{~nm})$ is then evaporated onto the wafer, followed by the deposition and patterning of a 6.5 - $\mu \mathrm{m}$-thick photoresist film (Microposit SJR 5740). The photoresist acts as a mold for electroplating a $2.5-\mu \mathrm{m}$-thick layer of gold that will serve as the electrodes and wiring (Fig. 3(b)). We electroplate with a commercial solution, Technic AR 434, at room temperature and 1 $\mathrm{mA} / \mathrm{cm}^{2}$. After stripping away the photoresist, the unplated regions of the seed layer is etched to electrically separate all the chips on the wafer by first dipping the wafer in a gold etchant followed by a quick dip in chrome etchant (Fig. 3(c)).
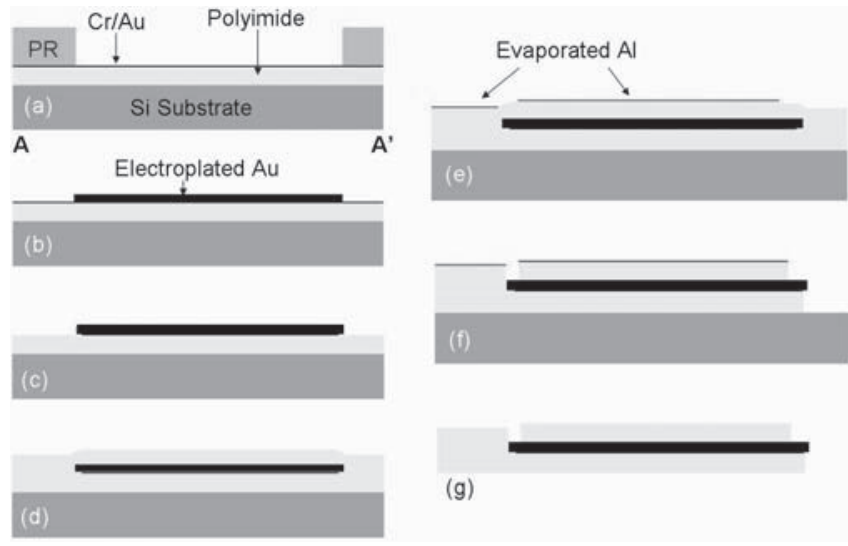

Fig. 3. Process flow for a micromachined electric-field probe.

We then spin on the second layer of polyimide, forming a sandwich of gold wires between two insulating polyimide layers (Fig. 3(d)). The second layer of polyimide is $13 \mu \mathrm{m}$ thick, in order 
to compensate for the topology of the wires and to guarantee good electrical insulation. We then mask the polyimide for an etch to expose the bondpads and tips on each probe as well as physically separate all the probe chips on the wafer from one another. The mask is a 50 -nm-thick evaporated aluminum film, which was patterned by lift-off (Fig. 3(e)). We etch the unmasked polyimide in a $100 \%$ oxygen plasma at 0.2 Torr in a reactive ion etcher (Oxford Instruments, Bristol, UK). The gold bondpads protect the layer of polyimide beneath them (Fig. 3(f)). Although we observe a little undercut of the probe tips, the majority of the polyimide beneath the exposed wire tips remains. This makes the probes onesided, that is, the electrodes face upwards and thus will only be able to measure the plasma on "top".

Finally, we peel the polyimide and gold "chips" off of the silicon substrate (Fig. 3(g)), and glue and wirebond them to the preamplification PCB. Figure 4 shows a probe chip mounted in the copper housing. To peel the polyimide structures off the wafer without damaging them, the adhesion promoter that we used (HD Microsystems VM-652) had to be applied in a particular pattern. If no promoter was used, the layer of polyimide would not adhere well to the wafer throughout the whole process. If too much promoter was used, the probes were impossible to remove without damaging them. Therefore, the solution we used was to apply the adhesion promoter to only the edges of the wafer before the first layer of polyimide was spun on. We did this by spinning the wafer at $300 \mathrm{rpm}$ and gently touching the wafer edge with a clean-wipe moistened with the adhesion promoter, resulting in ring of adhesion promoter on only the outer $5 \mathrm{~mm}$ or so of the wafer (Fig. 5) As long as no air bubbles got trapped underneath the polyimide layer after it had cured, the entire layer of polyimide would remain well attached to the wafer during the whole fabrication process. However, no adhesion promoter would be present to hold down the polyimide structures after the release etch.

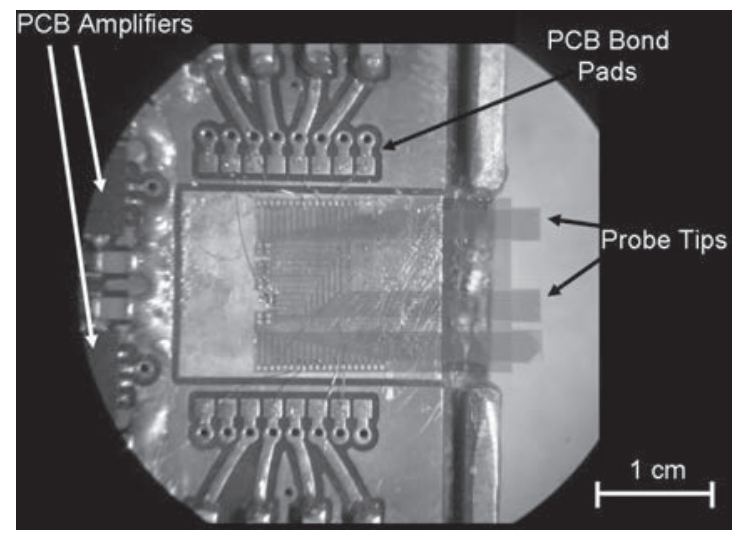

Fig. 4. Mounted probe housed in copper box.

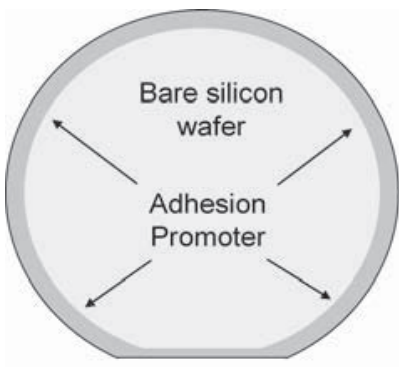

Fig. 5. Pattern of adhesion-promoter application

\section{EXPERIMENTAL RESULTS}

\section{A. Physical Properties}

The physical properties of the probe tips were investigated using a scanning electron microscope (Hitachi S4700 SEM). We found that after the oxygen plasma etch, some polyimide residue remained on the exposed gold pads and tips. This "microlace" made wirebonding difficult. To clean off the pads and tips, we etched the probe tips for five minutes in $15 \% \mathrm{CF}_{4}$ and $85 \% \mathrm{O}_{2}$, at the expense of a few hundred nanometers of gold. Figure 6 shows an SEM image of a released probe tip, and Fig. 7 shows an SEM of a set of $20-\mu \mathrm{m}$-wide wire tips separated by $20 \mu \mathrm{m}$.

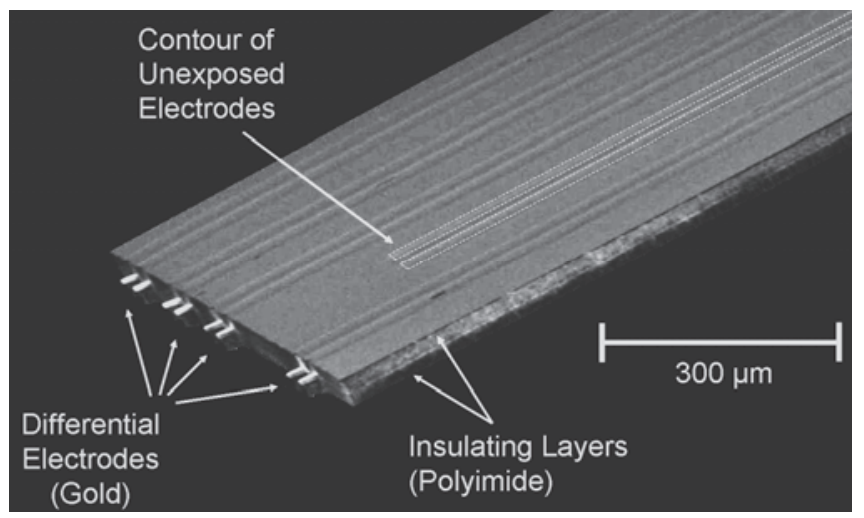

Fig. 6. SEM of probe shank and tips (both protruding and covered).

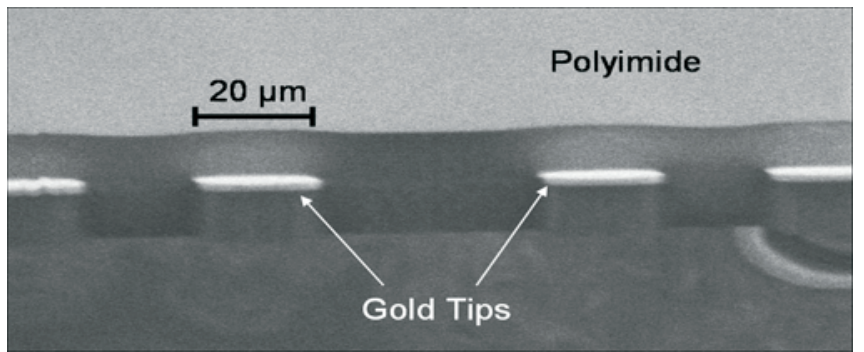

Fig. 7. SEM of protruding probe tips.

\section{B. Electrical Properties}

The measured tip-to-pad resistance was $22 \Omega$ for an $8-\mu \mathrm{m}$ wide tip and a $32-\mu \mathrm{m}$-wide wire. We characterized the frequency response of an assembled probe system (six functional probe tips, mounted on the PCB, and wired through the probe shaft to an external amplifier) by placing a short piece of wire protruding from a terminated $50-\Omega$ cable attached to a network analyzer (HP 8753D) near the probe tip array and capacitively coupling it to the probe tips; the results are shown in Fig. 8.

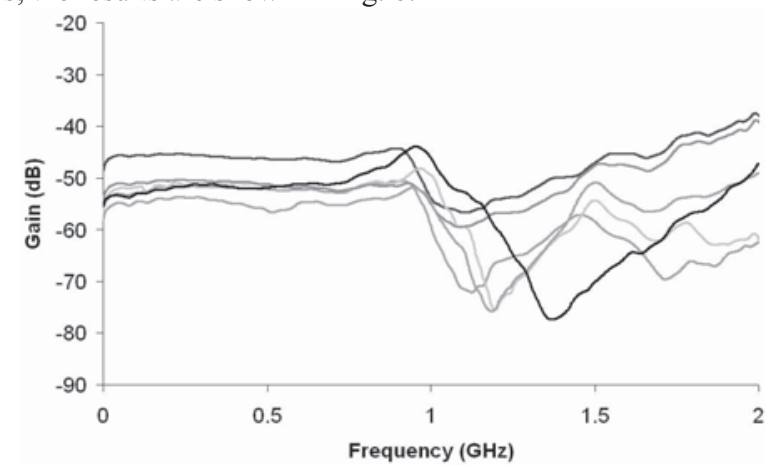

Fig. 8. Frequency-response plot for six protruding probe tips on a single probe shank.

The frequency response of the exposed probe tips have a relatively flat frequency response up to $1 \mathrm{GHz}$. Beyond $1 \mathrm{GHz}$, all probes display large fluctuations in the gain. This could be due to the inductance of the wire bonds and/or inadequate high-frequency printed-circuit-board layout, but is not associated with the probe tips themselves. For example, an interconnect inductance of $5 \mathrm{nH}$ with a circuit input capacitance of $5 \mathrm{pF}$ has a resonant frequency of 
about $1 \mathrm{GHz}$. We can conclude, therefore, that the stray capacitance is not too large.

Figure 9 shows the FFT of the smallest detectable signal magnitude by the complete system, found by connecting the device directly to a digital oscilloscope (LeCroy LT584) in a non-plasma environment. As can be seen, the electronics noise is far less than the detected signals with a peak amplitude of about $-20 \mathrm{dBm}$.

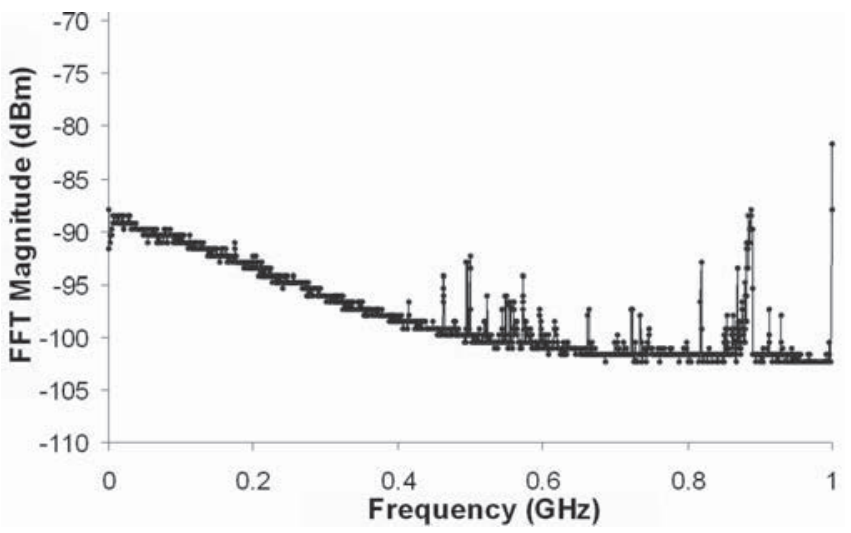

Fig. 9. Minimum detectable signal magnitude of complete system.

\section{Testing in Plasma Environment}

Initial testing of the probe in a plasma environment was done by inserting the assembled probe system (Fig. 10) into the LAPD. In one experiment, the tips are placed into a narrow current channel induced in the plasma. The results both demonstrate the functionality of the probes and reveal never-before-observed phenomena [7]. The physics behind the phenomena will be published separately in the future.

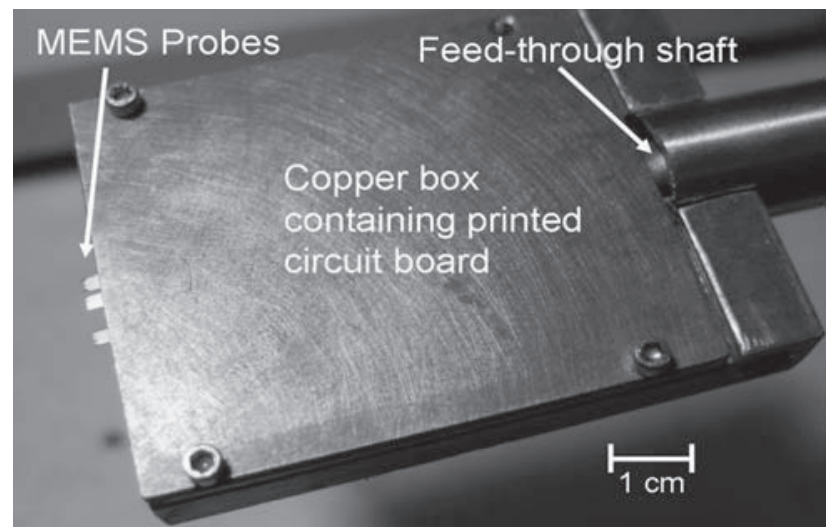

Fig. 10. Assembled probe system.

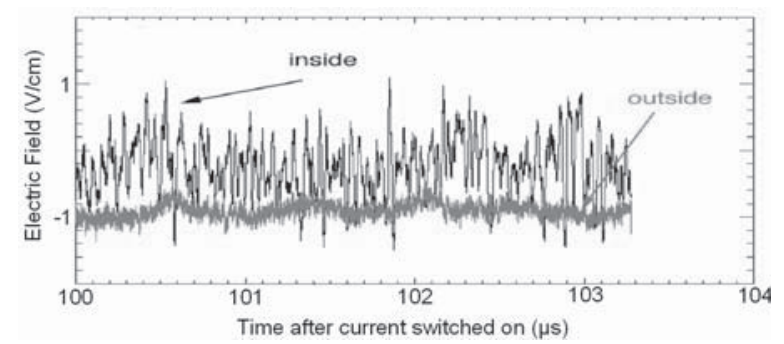

Fig. 11. Observed perpendicular electric-field activity inside and outside of the induced current sheet as a function of time.

Figure 11 plots the perpendicular electric field observed from both inside and outside of the current layer $100 \mu$ s after the current is switched on. The frequency spectrum of the signals plotted in Fig. 11 is shown in Fig. 12. Note the wave electric field is quite large, on the order of $1 \mathrm{~V} / \mathrm{cm}$, with the probes being small enough and close enough together to make this number believable.

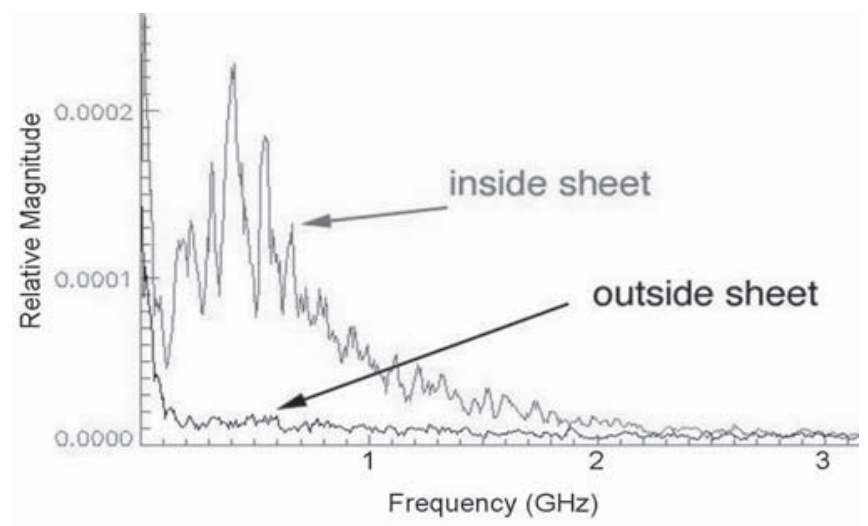

Fig. 12. Spectrum of perpendicular electric-field activity inside and outside of the induced current sheet.

\section{CONCLUSION}

We believe that there is great value in combining the controlled environment of laboratory-created plasma with the sophistication and precision of microprobes to further our understanding of fundamental plasma physics. We have demonstrated a first-generation microfabricated plasma probe that can take local measurements of the electric field in a magnetoplasma. It is clear from the initial tests done at the LAPD that there are many previously unobserved phenomena waiting to be characterized and explained. We are now working on improvements to these microprobes, as well as other types of diagnostics, such as a micrometer-scale ion-energy analyzer to measure ion-distribution functions. The ion-energy-analyzer design will be similar to that used by other researchers $[\mathbf{8}, \mathbf{9}]$, but scaled down in size. It will consist of three conductive grids biased in such a way that ions are filtered and collected based on their energies. Eventually, all of these plasma probes should incorporate on-chip amplifiers to convert the sub-picocoulomb charge levels to $50-\Omega$ signals.

\section{REFERENCES}

[1] I. H. Hutchinson, Principles of Plasma Diagnostics, $2^{\text {nd }}$ ed., 2002, Cambridge University Press.

[2] Bachet et al., "Laser-Induced Fluorescence Observation of Self-Organized Ion Structures Induced by Electrostatic Perturbations," Phys. Rev. Lett. vol. 80, no. 15, pp. 3260-3263 (1998).

[3] Sadeghi et al., "Transport of Argon Ions in an Inductively Coupled High-Density Plasma Reactor," Appl. Phys. Lett., vol. 70, no. 7, pp. 835-837 (1997).

[4] Kim et al., "An On-Wafer Probe Array for Measuring Two Dimensional Ion Flux Distributions in Plasma Reactors," Rev. Sci. Instr., vol. 73, no. 10, pp. 3494-3499 (2002).

[5] Freed et al., "Autonomous On-Wafer Sensors for Process Modeling, Diagnosis, and Control," IEEE Trans. Semicond. Manuf., vol. 14, no. 3, pp. 255-264 (2001).

[6] Wilson et al., "Silicon Micromachining using In Situ DC Microplasmas," J. Microelectromech. Syst., vol. 10, no. 1, pp. 50-54 (2001).

[7] Pribyl et al., "Debye-size Microprobes for Electric-Field Measurements in Laboratory Plasmas," Rev. Sci. Instr., (submitted Sept. 2005).

[8] Stone et al., "Instrument for Differential Ion Flux Vector Measurements On Spacelab 2," Rev. Sci. Instrum., vol. 56, no. 10, pp. 1897-1902 (1985).

[9] Blain et al., "High-Resolution Submicron Retarding Field Energy Analyzer for Low-Temperature Plasma Analysis," Appl. Phys. Lett., vol. 75, no. 25, pp. $3923-3925$ (1999). 